\title{
THE EFFECT OF RANDOM AGGREGATE FIELD ON SUPERCRITICAL CARBONATION OF CONCRETE
}

\author{
Xiaoxiong Zha ${ }^{1}$, Changchun Ren $^{2}$, Jiaqian Ning $^{3}$ \\ ${ }^{I}$ Department of Civil and Environmental Engineering, Harbin Institute of Technology, Shenzhen 518055, PR China \\ ${ }^{2}$ College of Urban Construction, Wuhan University of Science and Technology, Wuhan, 430065, PR China \\ ${ }^{3}$ Department of Civil and Environmental Engineering, Harbin Institute of Technology, Shenzhen 518055, PR China
}

\begin{abstract}
In order to study the supercritical carbonation process of concrete from the mesoscopic level, this paper is based on the theory of aggregate grading, by introducing a randomly distributed two-dimensional circular aggregate to simulate the supercritical carbonation process of concrete, the uneven carbonation boundary of concrete is obtained, and the carbonation degree of concrete is described by the maximum carbonation depth. The aggregate distribution of concrete is obtained by discussing the selection principle of the maximum particle size, the minimum particle size and the volume fraction of the aggregate; after the obtained aggregate distribution model is added to the supercritical carbonation model, the irregular boundary of concrete carbonation can be well simulated. The results show that the uneven distribution of aggregate in the cement mortar will lead to the non-uniformity of the carbonation depth of the concrete, which can be used to evaluate the effect of aggregate on the carbonation depth of the concrete.
\end{abstract}

Keywords: - Supercritical carbonation, Random aggregate, Carbonation depth

\section{INTRODUCTION}

The carbonation process of concrete consumes alkaline substances in concrete and destroys the alkaline environment in concrete, which leads to corrosion of steel bars and affects the durability of concrete[1]. Ashraf[2] reviewed the carbonation process of cement-based materials, indicating that carbonation may have a positive and negative impact on concrete performance. The carbonation reaction will change the porosity and pore size distribution in the concrete, affect the permeability, increase the compactness, and then affect the performance of the concrete[3][4]. In recent years, due to the rapid development of accelerated carbonation technology, the beneficial aspects of carbonation can be used to improve the performance of cement-based materials, M. FernándezBertos et al.[5] summarized the research and application of accelerated carbonation technology, and concluded that accelerated carbonation technology has great application prospects in the direction of waste solidification, and it is also a way to effectively absorb carbon dioxide, a greenhouse gas. The research[6] of Zha and Wang proved that the carbon storage capacity of different types of cement materials is different. Feng[7] used accelerated carbonation technology to transform the performance of cement bricks. In summary, further research is needed on the carbonation process of cement-based materials to maximize the positive effects of carbonation and reduce the negative effects of carbonation.

Carbonation of cement-based materials is a complex multiphysics process. The scholars of the former Soviet Union derived the theoretical model of natural carbonation of classical concrete by Fick's first law of diffusion. Anna V. Saetta et al. established differential equations between water vapor diffusion, thermal diffusion in concrete materials, and carbon dioxide diffusion, and applied finite element methods to solve this coupled nonlinear equations[8][9]. When the temperature and pressure exceed the critical values of carbon dioxide of $304.12 \mathrm{~K}$ and $7.38 \mathrm{MPa}$, the carbon dioxide is in a supercritical fluid state. At this time, carbon dioxide has a gas-like diffusion property and a liquid-like density, which is an excellent solvent. The carbon dioxide in this state has a low surface tension and can be easily diffused into a porous medium to accelerate the carbonation of the cement-based material. For the supercritical carbonation reaction, Zha et al. [10] established a mathematical model of supercritical carbonation with a pressure gradient as the main driving force under supercritical conditions. The model considers the chemical reaction, gas-liquid two-phase mass transfer and heat transfer, and compares the experimental data to verify the credibility of the model, which provides a basis for predicting the carbonation degree of cement-based materials in supercritical conditions under actual conditions.

Carbonation depth is one of the most important characteristics used to define the degree of carbonation reaction. In natural carbonation [11] and supercritical carbonation [10], the resulting carbonized regions are in an irregular shape and can be described by the maximum and minimum carbonation depths. However, in the current theoretical and numerical models, most of the concrete is considered to be an isotropic homogenous material, and the average carbonation depth is obtained instead of the 
maximum and minimum carbonation depth. However, in engineering applications, the maximum carbonation depth is required instead of the average carbonation depth. For example, the corrosion of steel bars in reinforced concrete requires the maximum carbonation depth to predict the state of the rusted steel bars. Concrete is a typical multiphase heterogeneous composite composed of cement mortar around the aggregate particles. It can be regarded as aggregate, cement mortar, interfacial transition zone and pore composition between aggregate and cement mortar, each phase is non-uniform [12], The randomly distributed aggregates are brought into a supercritical carbonation model, and the unevenness of the concrete will affect the distribution of carbonation depth.

Pan et al.[13] considering the random distribution of threedimensional aggregates in natural carbonation, found that despite the uniformity of cement mortar, the non-uniform distribution of aggregates in cement mortar will lead to macroscopic non-uniformity of concrete carbonation depth. Han et al.[14] obtained a random aggregation model based on CT imaging digital imaging method to study the effect of polymer on carbonation penetration. Huang et al.[15] and Ruan et al.[16] based on the meso-value concrete model, studied the influence of the distribution of coarse aggregate on the carbonation of concrete, and obtained a non-uniform carbonation depth. Yu et al. [17] studied the effect of twodimensional random porosity on the carbonation depth of supercritical carbonation of cement-based materials based on the elliptic autocorrelation function considering spatial correlation. It has been found that random porosity is suitable for supercritical carbonation of cement mortar and is not ideal for carbonation of concrete. In order to investigate the carbonation depth of supercritical carbonation of concrete, based on the existing supercritical carbonation numerical model[10], the random distribution of two-dimensional circular aggregate was introduced to study the influence of random aggregate on irregular carbonation depth.

\section{SUPERCRITICAL CARBONATION} THEORETICAL MODEL

The process of supercritical carbon dioxide carbonizing a cement substrate includes carbonation of carbon dioxide, dissolution and diffusion of carbon dioxide, gas-liquid twophase flow, and heat transfer. It can be described by chemical reaction rate, mass transfer equation, momentum transfer equation and energy transfer equation. The above physical and chemical processes are coupled to each other and affect the supercritical carbonation reaction process. The mathematical model of supercritical carbonation will be briefly described below.

\subsection{Governing Equations of Supercritical}

\section{Carbonation}

In combination with the supercritical carbonation process, considering the effects of dissolved carbon dioxide concentration, pressure, temperature, carbonation and other factors on the chemical reaction rate, the supercritical carbonation reaction control equations are established, see Eq. (1)-(4).

$$
\begin{aligned}
& \frac{\partial R_{c}}{\partial t}=\alpha_{1} \times f_{1}(h) \times f_{2}(g) \times f_{3}\left(R_{c}\right) \times f_{4}(T) \\
& \frac{\partial g}{\partial t}=\frac{\partial m_{C O_{2}}}{\partial t} \\
& \frac{\partial\left(n S_{\alpha} \rho_{\alpha}\right)}{\partial t}+\nabla \cdot\left(\rho_{\alpha} \mu_{\alpha}\right)=q_{\alpha} \\
& \mu_{\alpha}=-\frac{k k_{r \alpha}}{\mu_{\alpha}}\left(\nabla P_{\alpha}-\rho_{\alpha}^{g}\right) \\
& \left(\rho C_{q}\right)_{e f f} \frac{\partial T}{\partial t}=\nabla \cdot\left(k_{e f f} \nabla T\right)-\left(C_{g} \rho_{g} \mu_{g}+C_{w} \rho_{w} \mu_{w}\right) \nabla T
\end{aligned}
$$

In the formula, five independent variables are included, namely: carbonation degree $R_{c}$, carbon dioxide concentration $g$ in water, carbon dioxide pressure $P_{g}$, water pressure $P_{w}$, and temperature $T$. The detailed description of the parameters can be found in $\mathrm{Zha}[10]$.

\subsection{Initial Conditions and Boundary Conditions}

The above theoretical model is very complicated, and it is difficult to obtain its analytical solution, so it can only be numerically calculated. Therefore, the multiphysics finite element software COMSOL Multiphysics is used as the computing platform. The above five equations constitute the governing equations of supercritical carbonation, and the initial and boundary conditions of the corresponding independent variables are as follows:

Initial conditions:

$$
\begin{aligned}
& R_{c}=R_{c 0}=0 \text { and } \frac{\partial R_{c}}{\partial t}=0 ; P_{g}=P_{g 0} ; P_{w}=P_{w 0} \\
& g=g_{0}=0 \text { and } \frac{\partial g}{\partial t}=0 ; T=T_{0} \text { and } \frac{\partial T}{\partial t}=0 \text { at } t=0 \text { on } \Omega
\end{aligned}
$$

Boundary conditions:

$P_{g}$ and $P_{w}$ and $T$ are inflow boundaries, using the first type of boundary condition, namely the Dirichlet's conditions:

$$
P_{g}=\operatorname{Pt}(t), P_{w}=P_{w 0}, T=T t(t) \text { on } \Gamma_{1}
$$

The carbonation degree $R_{c}$ and the dissolved carbon dioxide concentration $g$ adopt the second type of boundary condition, namely Neumann's conditions: 
$\partial R_{c} / \partial n=0$ on $\Gamma_{2} \quad \partial g / \partial n=0$ on $\Gamma_{2}$

The detailed description of the parameters can be found in Zha[10].

\section{RANDOM FIELD MODEL OF AGGREGATE FOR CONCRETE}

Concrete is a typical multiphase heterogeneous composite composed of aggregate and cement mortar. The arrangement and distribution of aggregates are obviously random. Considering the aggregate as a particle combination structure, computer simulation can be used to generate a random aggregate structure similar to the prototype structure. The random aggregate model is used to analyze the microscopic scale of concrete materials, and then compensate for the defects of macroscopically using homogeneous materials to simulate concrete.

\subsection{Aggregate Grading Theory}

Aggregate grading is important for the performance of concrete. In order to guide the selection of aggregate grading in actual engineering, scholars from various countries have proposed many ideal grading curves. Zheng[18] gives the probability density function of concrete aggregate diameter in three-dimensional space:

$$
p_{3 D}(D)=\frac{n M^{n} D_{0}^{n}}{\left(M^{n}-1\right) D^{n+1}}
$$

Where $D$ represents the diameter of the aggregate, in the interval $\left[D_{0}, D_{m}\right]$. and $D_{0}$ are $D_{m}$ the minimum and maximum diameters of the aggregate, respectively. When $n=3.0$, it represents the equal volume aggregate distribution; when $n=2.5$, it represents the Fuller type aggregate distribution; the general aggregate distribution has $n$ values between (2.5, $3)$.

By integrating the probability density function, the Cumulative Distribution Function (CDF) of the aggregate can be obtained. Any one of them corresponds to the aggregate passage rate of the corresponding sieve hole, that is, the ratio of the aggregate passing through the aperture to the total aggregate. It should be noted that the CDF is for certain properties of the aggregate, such as the diameter of the aggregate. In simulating the random aggregate distribution of concrete, $\mathrm{Li}[19]$ derived the aggregate distribution function of aggregate diameter of mediumvolume aggregate distribution in three-dimensional space based on Eq. (8):

$$
P_{3 D}(D)=\frac{M^{3} D_{0}^{3}}{\left(M^{3}-1\right)}\left(\frac{1}{D_{0}^{3}}-\frac{1}{D^{3}}\right)
$$

In this paper, the supercritical carbonation process of concrete is simulated in a two-dimensional plane, and the random aggregate adopts a two-dimensional circular shape.Based on the geometric probability relationship, the cumulative distribution function of the circular diameter of the two-dimensional section is derived by using the cumulative distribution function of the aggregate diameter in three dimensions[19]:

$$
P_{2 d}(d)=1-\frac{2 M^{2} D_{0}^{2}}{3\left(M^{2}-1\right) d^{2}}\left(1-\frac{d^{2}}{D_{m}^{2}}\right)^{1.5}
$$

Where $\mathrm{d}$ is the diameter of the section circle, $0 \leq d \leq D_{m}$.

In order to meet the actual situation of the particle size distribution and to facilitate calculation, the minimum particle size is introduced, and $D_{0} \leq d \leq D_{m}$ is obtained, and the corresponding cumulative distribution function is converted into $P_{2 d}^{\prime}(d)$ :

$$
P_{2 d}^{\prime}(d)=\frac{d^{2}\left(D_{m}^{2}-D_{0}^{2}\right)^{1.5}-D_{0}^{2}\left(D_{m}^{2}-d^{2}\right)^{1.5}}{d^{2}\left(D_{m}^{2}-D_{0}^{2}\right)^{1.5}}
$$

\subsection{Aggregate Generation}

Based on the circular diameter accumulation distribution function of the two-dimensional section, a two-dimensional circular aggregate geometric model that satisfies aggregate gradation can be randomly generated in MATLAB according to the algorithm shown in Fig -1 .

In the above algorithm, for the process of "Position judgement", the requirements are: (1) The aggregate generated must locate in the calculation domain; (2) The overlap between the newly formed circular aggregate and the existing circular aggregate is judged. If the requirements are not met, the next step is to return and regenerate the aggregate position.

According to the above algorithm, the simulation shown in Fig -2 is performed. A random aggregate model with a $40 \%$ aggregate area percentage is generated in a plane of $100 \times 100 \mathrm{~mm}$.Among them, the maximum particle size of aggregate is $15 \mathrm{~mm}$, the minimum particle size is $4 \mathrm{~mm}$, and the number of aggregates generated by simulation is 96 . 


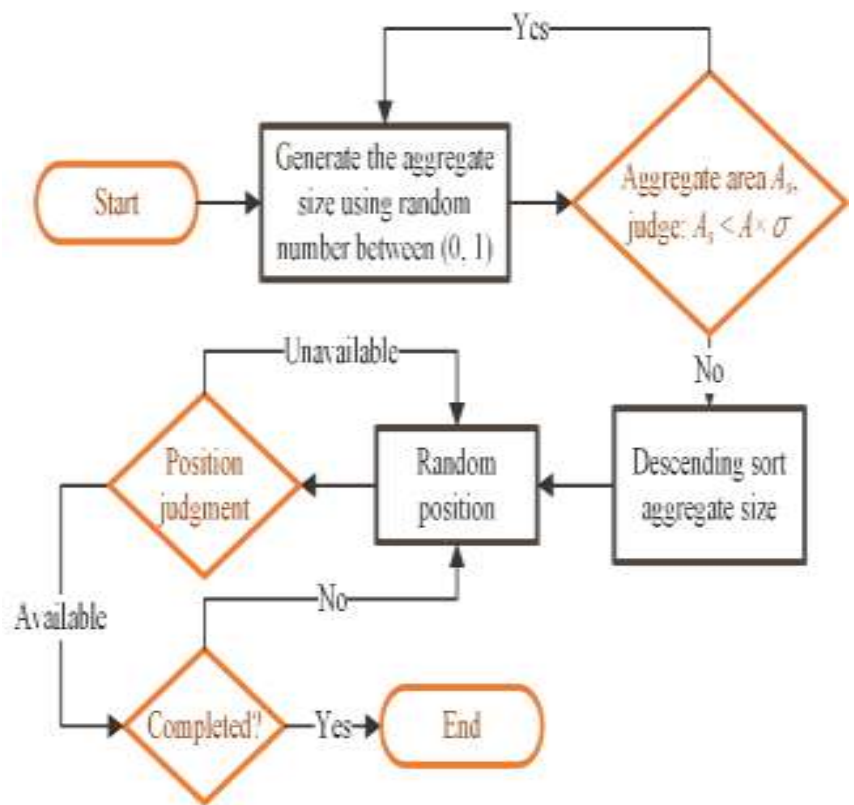

Fig -1: Flowchart of Random Aggregate generation

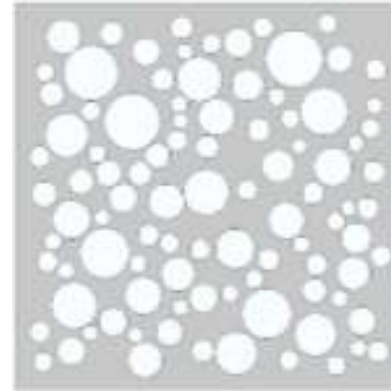

Fig -2: Examples of two dimensional analog circular aggregate

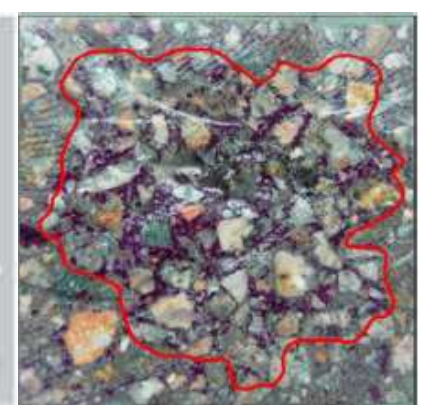

Fig -3: Concrete block[10]

\section{EFFECTS OF RANDOM AGGREGATE} MODELLING ON THE SUPERCRITICAL CARBONATION PROCESS IN CONCRETE

According to the supercritical carbonation test in the literature of $\mathrm{Zha}[10]$ and $\mathrm{Yu}[17], \quad \mathrm{Fig}-3$ shows the supercritical carbonation zone of concrete. The dimensions of the concrete cube are both $100 \times 100 \times 100 \mathrm{~mm}$. The concrete cube reacted for $5.8 \mathrm{~h}$ under supercritical carbon dioxide. It can be clearly seen from Fig. 3 that the noncarbonized area around the center of the concrete test block has irregular boundaries.

In order to simulate the carbonation process shown in Fig -3, the two-dimensional supercritical carbonation model proposed by Zha[10] was used for numerical simulation. The geometric model in the supercritical carbonation model of concrete uses the algorithm based on Fig -1 to generate a random aggregate distribution model to simulate the heterogeneity of concrete. The concrete cube has a crosssectional dimension of $100 \times 100 \mathrm{~mm}$, wherein the concrete aggregate area percentage is $40 \%$, the maximum particle size is $15 \mathrm{~mm}$, and the minimum particle size is $4 \mathrm{~mm}$. The average porosity $\varepsilon_{m}$ of concrete is 0.122 , and the intrinsic permeability coefficient $k_{0}$ is $3 \times 10^{-21} \mathrm{~m}^{2}$ [21].

This paper compares the carbonation results generated by COMSOL simulation with the existing experimental results[10][20]. In this paper, the geometric model of the supercritical carbonation model is first derived from the random aggregate model obtained in the third part. A random aggregate model was obtained in MATLAB by the given area percentage of the aggregate and the maximum and minimum particle sizes of the aggregate size, and then saved as a mat file and imported into the supercritical carbonized COMSOL model. The geometric model of the supercritical carbonation model at this time is composed of random aggregates.

According to the above supercritical carbonation model, the results of four kinds of random aggregate distribution concrete blocks after carbonation for 5.8 hours under supercritical conditions were calculated and compared with the results of two-dimensional model of homogeneous concrete. The random aggregate distribution model is shown in Fig -4. Fig -4 shows a random aggregate distribution model based on a $40 \%$ aggregate area, a maximum particle size of $15 \mathrm{~mm}$, and a minimum particle size of $4 \mathrm{~mm}$. The total number of aggregates simulated in Sample 1, Sample 2, Sample 3, and Sample 4 are 119, 106, 96, and 114, respectively. The concrete homogenization two-dimensional geometric model is shown in Fig -5.

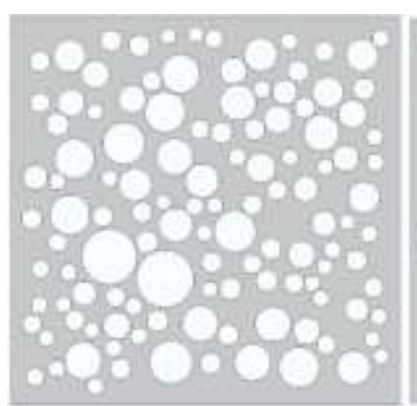

(1) Sample 1

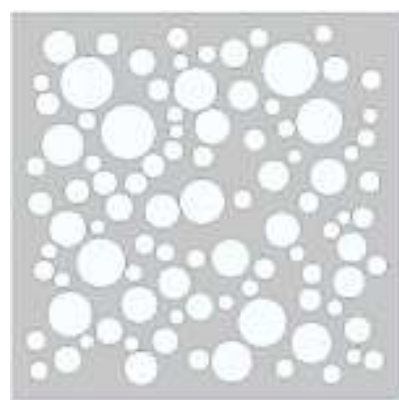

(3) Sample 3

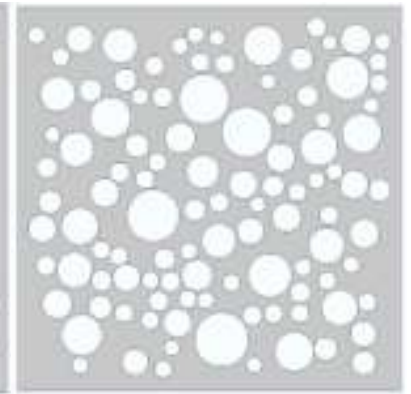

(2) Sample 2

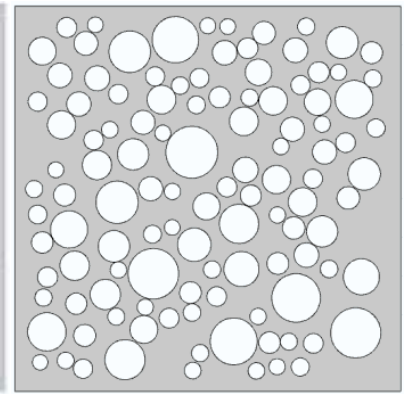

(4) Sample 4
Fig -4: Geometric model of random aggregate 


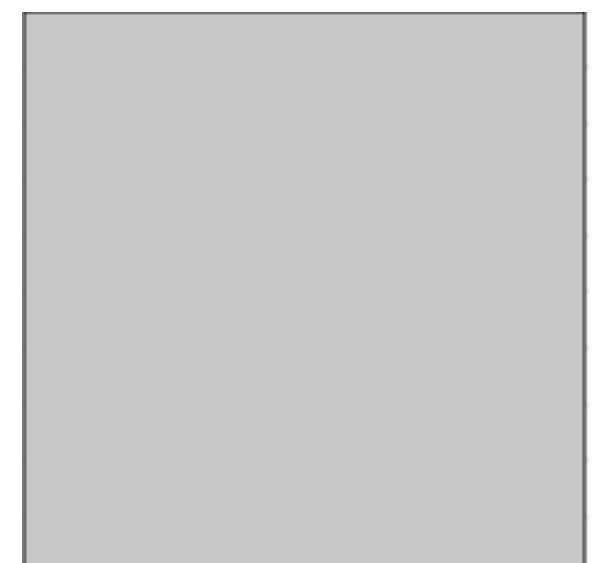

Fig -5: Uniform concrete geometric model

Fig-6 shows the carbonation curve of the concrete test block section calculated based on the supercritical carbonation model of random aggregate. The area inside the yellow curve indicates the non-carbonized area. Fig -7 shows the carbonation curve obtained by using concrete as a homogeneous material. Table -1 shows a detailed comparison of the average carbonation depth and maximum carbonation depth obtained from Fig -6 and Fig-7.

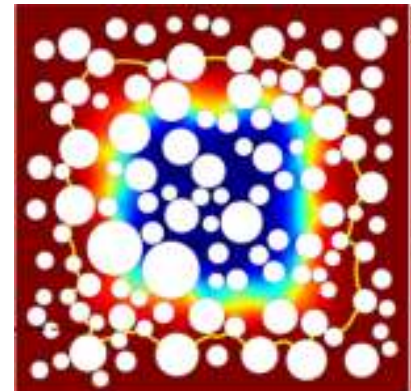

(1) Sample 1

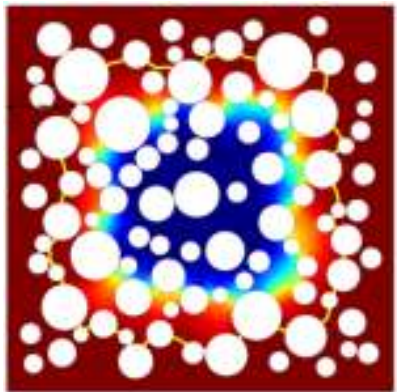

(3) Sample 3

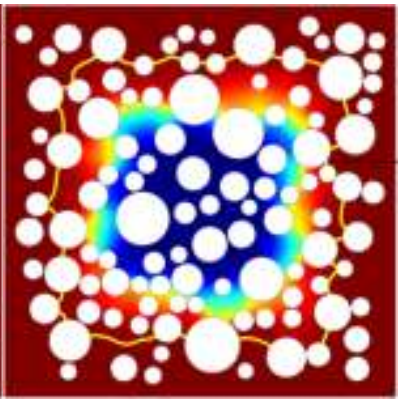

(2) Sample 2

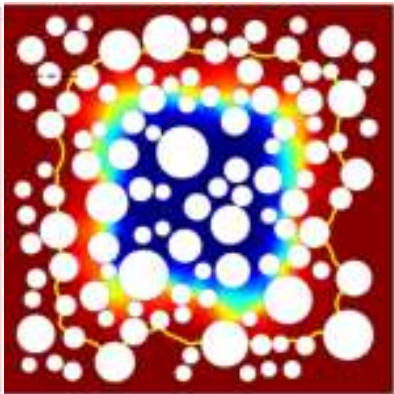

(4) Sample 4
Fig -6: Random aggregate model and carbonation results of concrete

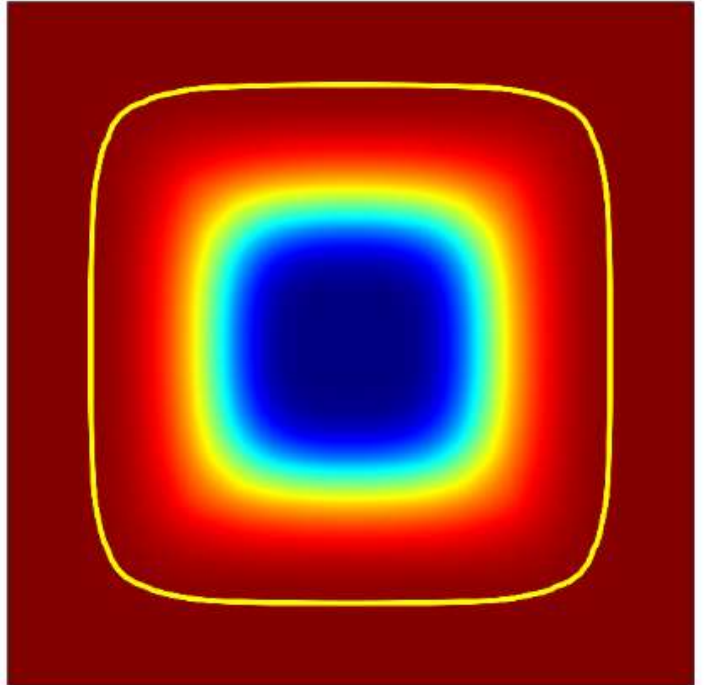

Fig -7: Uniform concrete model and carbonation results of concrete

Table -1: The average and maximum carbonation depth of concrete

\begin{tabular}{|c|c|c|c|c|c|c|}
\hline \multirow{3}{*}{$\begin{array}{ll}\text { Carbonatio } \\
n & \text { depth } \\
(\mathrm{mm}) & \end{array}$} & \multirow{3}{*}{ Test $[10]$} & \multicolumn{5}{|c|}{ Numerical simulation } \\
\hline & & \multicolumn{4}{|c|}{ Random aggregate model } & \multirow{2}{*}{$\begin{array}{l}\text { Uniform } \\
\text { concrete } \\
\text { mode }\end{array}$} \\
\hline & & 1 & 2 & 3 & 4 & \\
\hline ave & 25 & 16.4 & 15.3 & 15.8 & 15.7 & 12.7 \\
\hline Iaximum & 7.0 & 20.2 & 19.2 & 18.9 & 19.4 & 12.7 \\
\hline
\end{tabular}

From Fig -6, because of the uneven distribution of aggregates, the irregularity of the carbonation curve of the concrete test block section is caused. It can be seen that the carbonation results obtained by different random aggregate models generated based on the algorithm and parameters described in Section 3.2 are similar. This shows that the simulation of concrete carbonation depth by different random aggregate models is not much different. This shows the rationality of the algorithm.

Comparing the experimental results[10], in terms of the maximum carbonation depth, the results obtained by different random aggregate models have a certain randomness, and there is no rule to follow. The average value of the results was $19.43 \mathrm{~mm}$, and the deviation rate was $28 \%$. The result of the Uniform concrete model was $12.7 \mathrm{~mm}$, and the deviation rate was $52.96 \%$. Therefore, the random aggregate model has great advantages, and its simulation results are close to the experimental data, which can effectively guide the application in engineering practice.

In terms of the average carbonation depth, the simulation results of the random aggregate model have large deviations. The reasons are as follows: (1) The random aggregate model used in this paper is circular and has certain limitations, which is deviated from the shape and size of the actual aggregate; (2) The random aggregate model is randomly generated by the algorithm. For the specific experimental results, not all random aggregate models are applicable, and 
more parameters need to be introduced later to optimize the algorithm. In addition, the results of the random aggregate model are larger than the Uniform concrete model results. The reason is that in the supercritical carbonation model, the introduction of aggregates reduces the cement content per unit volume of concrete. This reduces the amount of carbon dioxide absorbed per unit volume of cement-based material, which in turn leads to a further deepening of the carbonation reaction of the concrete material.

Compared to considering concrete as a homogeneous material, the introduction of a random distribution of aggregates can improve the prediction of the maximum carbonation depth. At the same time, in the simulation results, it was found that the existence of aggregates, the development of carbonation depth has a certain delay, indicating that the aggregate has a hysteresis effect on the carbonation reaction. The reason may be that the impermeability of the aggregate hinders the diffusion of carbon dioxide. As the carbonation reaction continues, the carbon dioxide must flow around the surface of the aggregate.

\section{CONCLUSION}

In this paper, a random generation method of twodimensional circular aggregate is established based on the cumulative distribution function of two-dimensional aggregate. The meso-concrete geometry model considering concrete inhomogeneity is obtained and applied to the supercritical theoretical model of concrete. By comparing the experimental data with the numerical results, the accuracy of the improved model is studied and the following conclusions are obtained:

(1) After introducing the random aggregate model, the irregular carbonation curve of the concrete section is obtained. At the same time, it has higher accuracy in predicting the maximum carbonation depth of concrete.

(2) For the random aggregate model, when the percentage of aggregate area and the maximum and minimum particle sizes of the aggregate are determined, the difference between the supercritical carbonation calculation results of the different aggregate random models is small.

The feasibility of the random aggregate model was verified. (3) The supercritical carbonation simulation using a circular random aggregate model has a poor effect in simulating the average carbonation depth.

This shows that the circular aggregate has certain limitations on the simulation of the actual aggregate. It is necessary to continue to optimize and study the shape and size of the random aggregate model.

(4) According to the results of the random aggregate model, it is found that there is hysteresis in the carbonation process around the aggregate, and the aggregate has a certain hindrance to the carbonation of concrete.

\section{ACKNOWLEDGEMENTS}

This study and the completion of this paper were supported by the National Nature Science Foundation of China (No. 51578181), Natural Science Foundation of Guangdong Province (No. 2016A030313665) and Shenzhen Science and Technology Plan Project (No. JCYJ20150327155221857). The authors would like to extend their appreciation to Shenzhen Carbon Storage Cement-based Materials Engineering Laboratory.

\section{REFERENCES}

[1] PapadakisVG , VayenasCG , Fardis MN. Experimental investigation and mathematical modeling of the concrete carbonation problem[J]. Chemical Engineering Science, 1991, 46 : 13331338 .

[2] W. Ashraf. Carbonation of cement-based materials: challenges and opportunities[J], Constr. Build. Mater. 120 (2016) 558-570.

[3] J. Han, G. Pan, W. Sun, et al. Application of nanoindentation to investigate chemomechanical properties change of cement paste in the carbonation reaction, Sci. China Technol. Sci. 55 (3) (2012) 616622.

[4] TiejunZhao,Shujin Li. Effect of carbonation on permeability and porosity of concrete[J]. Industrial Construction, 2003, 33(1): 46-47(in Chinese).

[5] M. FernándezBertos, S.J.R. Simons, C.D. Hills. A review of accelerated carbonation technology in the treatment of cement-based materials and sequestration of $\mathrm{CO} 2[\mathrm{~J}]$. Journal of Hazardous Materials, 2004, B112 : 193-205.

[6] XiaoxiongZha, Haiyang Wang, GanlinFeng. Effects of supercritical carbonation on the property and pore structure of cement-based materials [J]. Journal of Harbin Institute of Technology, 2014, 46(11):5255(in Chinese).

[7] GanlinFeng. Experimental and numerical study of accelerated carbonation in cement brick and tile [D]. Harbin Institute of Technology, 2013(in Chinese).

[8] A. V. Saetta, B. A. Schrefler and R.V. Vitaliani. The carbonation of concrete and the mechanism of moisture, Heat and Carbon Dioxide Flow through Porous Materials[J]. Cement and Concrete Research , 1993, 23(4) : 761-772.

[9] A. V. Saetta, B. A. Schrefler and R.V. Vitaliani. 2-D Model for Carbonation andMoisture/Heat Flow in Porus Materials[J]. Cement and Concrete Research, 1995 25(8) : 1703-1712.

[10] XiaoxiongZha, MinYu , JianqiaoYe, et al. Numerical modeling of supercritical carbonation process in cement-based materials $[\mathrm{J}]$. Cement and Concrete Research, 2015, $72: 10-20$.

[11] K.J. Beasley, Carbon dating concrete cracks, J. Perform. Constr. Facil. 29(025140021) (2015).

[12] Chandra S, Berntsson L. 6-Lightweight Aggregate Concrete $\quad$ Microstructure[M]//Lightweight 
Aggregate Concrete. Norwich , NY : William Andrew Publishing, 2002 : 131-166.

[13] Zichao Pan, Xin Ruan, Airong Chen. Numerical simulation of concrete carbonation process on mesoscale[J]. Tongji Univ. (Nat. Sci.) 40 (6) (2012) 900905 (in Chinese).

[14] J. Han, W. Liu, S. Wang, et al., Effects of crack and itz and aggregate on carbonation penetration based on 3D micro X-ray $\mathrm{Ct}$ microstructure evolution, Constr. Build. Mater. 128 (2016) 256-271.

[15] Q. Huang, Z. Jiang, W. Zhang, et al., Numerical analysis of the effect of coarse aggregate distribution on concrete carbonation, Constr. Build. Mater. 37 (2012) 27-35.

[16] X. Ruan, Z. Pan, Mesoscopic simulation method of concrete carbonation process, Struct. Infrastruct. Eng. 8 (2) (2012) 99-110.

[17] Min Yu, HaoBao, JianqiaoYe, et al. The effect of random porosity field on supercritical carbonation of cement-based materials[J]. Construction and Building Materials, 2017, $146: 144-155$.

[18] Zheng J J. Mesostructure of concrete-stereological analysis and some mechanicalimplications [M] . Delft : Delft University Press, 2000.

[19] XuemeiLi. Thetwo-dimensionalcomputersimulationandstudyofwalleffect for mesoscopic structure of concrete[D]. Northern Jiaotong University,2002 (in Chinese).

[20] ComsolMultiphysics. ComsolMultiphysics User Guide (Version 4.3a) 2012.

[21] Baroghel B V, Thiéry M, Wang X. Modelling of Isothermal Coupled Moisture-Ion Transport in Cementitious Materials[J]. Cement and Concrete Research, 2011, 41(8): 828-841.

\section{BIOGRAPHIES}

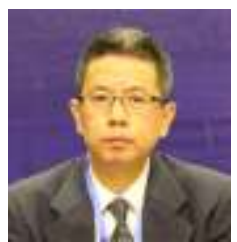

Prof.XiaoxiongZha, Civil \& Environmental Engineering, Harbin Institute of Technology, Shenzhen; 7th, 8th President of China Steel Construction Society Association for Steel-Concrete Composite Structures (CSCS-ASCCS);

Director of Shenzhen Carbon Storage Cement-based Materials Engineering Laboratory; Research Interests: Carbon Capture \& storage, Cement-free Geopolymer Concrete, Prefabricated Modular Building, Concrete-filled Steel Tube, etc.

Homepage: http://www.zhahero.icoc.cc/

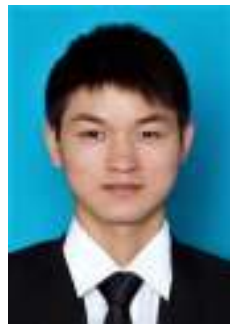

MscChangchun Ren, Urban Construction, Wuhan University of Science and Technology.

Research Area: The Application of Random FieldOn Supercritical Carbonation
PHD Candidate Jiaqian Ning, Civil \& Environmental Engineering, Harbin Institute of Technology, Shenzhen.

Research Area: CO2 Recovery \& Its Application In Construction Materials 